\title{
EPIDEMIOLOGIA, FATORES CLIMÁTICOS E DISTRIBUIÇÃO ESPACIAL DA DENGUE EM UMA CAPITAL DO NORDESTE DO BRASIL
}

\author{
SANTOS JÚNIOR, Claudio José dos - claudiosantos_al@hotmail.com \\ Universidade Estadual de Ciências da Saúde de Alagoas - UNCISAL
}

SILVA, Jackson Pinto - jacksonpinto@bol.com.br
Instituto Federal de Alagoas - IFAL

\begin{abstract}
RESUMO: Este trabalho teve como objetivos avaliar a correlação de parâmetros meteorológicos com a incidência de dengue em Maceió-AL, Brasil, e descrever a distribuição espacial e perfil epidemiológico. Foram considerados os registros de casos novos de 01/01/2010 a 31/12/2016. A incidência foi verificada através da Taxa Anual Média de Incidência (TAM). A correlação de Pearson foi utilizada para avaliar a relação entre fatores climáticos e incidência de casos. A espacialização foi realizada por meio da geração de mapas temáticos. Como resultados verificou-se que, na estratificação da TAM, a capital apresentou $57 \%$ do período em análise com alta incidência e $29 \%$ do intervalo temporal com característica de epidemia. Verificou-se que nenhum bairro apresentou ausência da doença na totalidade período em análise. As áreas consideradas de "média incidência" se distribuíram ao longo de todo o território da capital, enquanto as de "alta incidência" encontravam-se concentradas, principalmente, nas regiões Sul, Noroeste e Norte. O município apresentou somente dois bairros com "baixa incidência". $\mathrm{Na}$ análise de densidade de casos, verificou-se alta densidade e formação clusteres em três regiões do município e grande concentração de ocorrência de dengue nas porções Sul e no Litoral. Houve correlação de forte a moderada entre a incidência de dengue e as variáveis umidade e precipitação pluviométrica em $60 \%$ e $70 \%$ do período analisado, respectivamente. A pesquisa apontou que as variáveis precipitação e umidade influenciam na epidemiologia da dengue, além de evidenciar a existência de áreas prioritárias de intervenção no combate da doença.
\end{abstract}

Palavras-Chave: Dengue; epidemiologia; clima; Alagoas; Brasil.

\section{EPIDEMIOLOGY, CLIMATIC FACTORS AND SPATIAL DISTRIBUTION OF DENGUE IN A CAPITAL OF NORTHEAST BRAZIL}

ABSTRACT: This study aimed to evaluate the correlation of meteorological parameters with dengue incidence in Maceió-AL, Brazil, and to describe the spatial distribution and epidemiological profile. We considered the records of new cases from 01/01/2010 to $12 / 31 / 2016$. The incidence was verified through the Average Annual Incidence Rate (TAM). Pearson's correlation was used to assess the relationship between climatic factors and case incidence. Spatialization was performed through the generation of thematic maps. As results, it was found that, in the stratification of TAM, the capital presented $57 \%$ of the period under analysis with high incidence and $29 \%$ of the time interval with characteristic of epidemic. It was found that no neighborhood had absence of the disease in the entire period under analysis. The areas considered of "medium incidence" were distributed throughout the territory of the capital, while those of "high incidence" were concentrated mainly in the South, Northwest and North. The municipality presented only two neighborhoods with "low incidence". In the density analysis of cases, it was verified high density and clusters formation in three regions of the municipality and high concentration of dengue occurrence in the southern and coastal portions. There was a strong to moderate correlation between the incidence of dengue and the variables humidity and rainfall in $60 \%$ and $70 \%$ of the analyzed period, respectively. The research pointed out that the variables precipitation and humidity influence the epidemiology of dengue, as well as highlighting the existence of priority areas of intervention to combat the disease.

Keywords: Dengue; epidemiology; climate; Alagoas; Brazil. 


\section{INTRODUÇÃO}

A dengue é uma doença infecciosa febril aguda, de notificação compulsória, causada por um arbovírus da família Flaviridae e é transmitida, no Brasil, por meio do mosquito Aedes aegypti infectado pelo vírus do dengue (DENV) (LOPES et al., 2018). Atualmente, a dengue é considerada, nacionalmente e em diversos países do mundo, como sendo um dos principais problemas de saúde pública a ser enfrentado pelos serviços de saúde (WHO, 2012; BHATT et al., 2013). A infecção pelo DENV causa uma doença de amplo espectro clínico, incluindo de formas pouco sintomática até quadros que podem evoluir para o óbito (LOPES et al., 2018).

A doença está amplamente distribuída no Brasil pelo fato de o vetor ser encontrado em todas as regiões do país e de sua proliferação ser facilitada pelo clima quente e úmido predominante na maior parte do território nacional (AULT et al., 2019). Por seu caráter endêmico e epidêmico de grande magnitude e uma vez que parte significativa dos indivíduos acometidos está em idade produtiva, a dengue tem importante impacto social e econômico (HONORATO et al., 2014).

A circulação simultânea de três sorotipos do vírus do dengue (DENV-1 DENV-2 e DENV-3) no Brasil, a partir do ano 2000, e mais recentemente, a reintrodução do DENV-4 têm contribuído para o agravamento da situação epidemiológica dessa doença e para comportamentos antes não conhecidos (BARRETO; TEIXEIRA, 2008; RAMOS-CASTANEDA et al., 2017)

Sabe-se que a epidemiologia, como área de estudo, possibilita o melhor manejo do processo saúde-doença e que, ao mesmo, tempo, a identificação da caracterização das áreas de ocorrência de uma doença é um dos pilares dos estudos ecológicos, bem como da epidemiologia social (RODRIGUES, 2014).

O catálogo dos ambientes com maiores taxas de prevalência e incidência e a identificação do perfil dos acometidos permitem o planejamento de ações preventivas de saúde e subsidiam a formulação de estratégias e políticas públicas de promoção de saúde nos ambientes de maior concentração dos casos de uma patologia (RODRIGUES, 2014). A análise espacial, por sua vez, é utilizada há muitos anos para verificar variação de características geoambientais regionalizados. Atualmente, devido à re-emergência de muitas doenças infecciosas e parasitárias, bem como às frequentes epidemias em diversas regiões do país, vem sendo aplicada para o estudo de padrões espaciais da incidência e mortalidade por diversas doenças (CAVALCANTE et al., 2013).

Dessa maneira, o objetivo do presente trabalho consistiu na realização de levantamento e de mapeamento da situação epidemiológica da dengue no município de Maceió, capital do estado de Alagoas, verificando-se, pois, a correlação de tais dados com os parâmetros meteorológicos da região.

Tais informações contribuirão para que os órgãos de saúde da Administração Pública possam identificar as áreas que concentram as maiores taxas de ocorrência dessa doença, além de possibilitar a identificação e a caracterização de fatores associados à ocorrência dessa patologia, viabilizando a adoção de ações de saúde, assistência direcionada às comunidades, combate ao agente transmissor e indicação de áreas prioritárias para implementação de medidas de controle. 


\section{MATERIAL E MÉTODO}

Trata-se de um estudo ecológico dos indicadores de dengue mediante análise temporo-espacial sobre o período de 2010 a 2016. A área de estudo foi o município de Maceió, capital do estado de Alagoas, Brasil, e suas divisões administrativas de referência (bairros).

Foram utilizados dados de notificações de casos novos de dengue por município de residência, obtidos através da Secretária Municipal de Saúde de Maceió (SMS) e extraídos do Sistema Nacional de Agravos de Notificação (SINAN).

Foram incluídos todos os casos novos de dengue com notificação no período de 2010 a 2016 e investigação diagnóstica concluída como "caso confirmado" em residentes do município de Maceió-AL.

Os dados de população utilizados neste trabalho são referentes ao censo populacional realizado em 2010 pelo Instituto Brasileiro de Geografia e Estatística (IBGE) e foram extraídos do site do órgão (www.ibge.gov.br).

Os dados climáticos, por sua vez, foram disponibilizados pelo Instituto Nacional de Meteorologia (INMET) e se referem às médias mensais dos parâmetros meteorológicos (temperatura, umidade relativa do ar e precipitação) registrados para o período da pesquisa e obtidos pela estação meteorológica de superfície do INMET no 82994 (Maceió-AL; Latitude: -9.66; Longitude: -35.7).

Para a definição das áreas de risco e da distribuição espacial de casos de dengue, empregou-se o cálculo da Taxa Anual Média de Incidência (TAM), calculada para o Município e por bairro, conforme metodologia preconizada pelo Ministério da Saúde (2002).

$\mathrm{Na}$ estratificação espacial da TAM, o município e seus bairros foram categorizados segundo os critérios da Organização Mundial e do Programa Nacional de Controle de Dengue: baixa incidência (até 100 casos/100 mil habitantes); média incidência (100 a 300 casos/100 mil habitantes); alta incidência (300-1000 casos/100 mil habitantes) e epidêmicos (>1000 casos/100 mil habitantes) (MINISTÉRIO DA SAÚDE, 2002; WHO, 2012).

Tendo em vista que o município de Maceió possui um grande número de bairros com pequenas áreas, fez-se necessário realizar uma suavização das taxas de incidência através da abordagem do estimador bayesiano empírico local. A aplicação desse método justifica-se, pois, na metodologia de cálculo tradicional, as áreas que possuem denominadores (população) incipientes, têm grande chance de ter sua realidade mascarada. Segundo Honorato et al. (2014) a abordagem bayesiana aproxima as taxas brutas das pequenas áreas em direção a taxa média de seus vizinhos, permitindo análises mais informativas e estáveis.

A realização dos cálculos de estatística espacial foi construída por meio do software livre TerraView 4.4.2 e para a realização da geração dos mapas temáticos empregou-se o programa Quantum GIS 3.0. A base cartográfica do município foi obtida junto ao Instituto Brasileiro de Geografia e Estatística (IBGE) e levou em consideração o sistema Mercator Transversal Universal e o DATUM SIRGAS 2000. Para avaliar o padrão de distribuição de casos, utilizou-se o método de estimação de curvas de densidades de Kernel por meio da 
elaboração de mapas de calor, ferramenta empregada para estimar a intensidade do evento em uma região, mesmo em áreas onde o processo não tenha ocorrido ou que tenham demonstrado ocorrência real.

A correlação de Pearson ( $r$ ) foi utilizada para analisar a associação entre a incidência de dengue e os elementos climáticos: temperatura média, pluviosidade e umidade relativa do ar. Adotou-se como variável dependente $(Y)$ os valores de incidência da doença e como independente $(X)$ os dados meteorológicos, além da intepretação de Figueiredo e Silva (2009) que expõe: se $r=0,9$ para mais ou para menos indica uma correlação muito forte; $r=0,7$ a 0,9 positivo ou negativo indica uma correlação forte; $r=0,5$ a 0,7 positivo ou negativo indica uma correlação moderada; $r=0,3$ a 0,5 positivo ou negativo indica uma correlação fraca; $r=0$ a 0,3 positivo ou negativo indica uma correlação desprezível. Esses dados foram analisados utilizando-se os programas Microsoft Excel e BioEstat 5.3. Foi adotado um nível de significância estatística de $95 \%(p<0,05)$.

Por se tratar de estudo realizado com o uso de dados oriundos de fontes secundárias, não foi necessária a aprovação pelo Comitê de Ética em Pesquisa.

\section{RESULTADOS}

A dengue se comportou de maneira distinta na cidade de Maceió-AL entre o intervalo que compreende os anos de 2010 e 2016. A capital, que é composta por 50 bairros, chegou a apresentar 14.824 casos em 2010 e 2.708 em 2011, totalizando 48.094 diagnósticos confirmados no período de 2010 a 2016 .

Tabela 1 - Número de casos e coeficiente de incidência de dengue no Município de Maceió-AL. Brasil, 2010 a 2016.

\begin{tabular}{|c|c|c|c|c|c|c|c|c|c|c|c|c|c|c|c|}
\hline \multirow[b]{2}{*}{ Ano/Mês } & \multirow[b]{2}{*}{ Jan } & \multirow[b]{2}{*}{ Fev } & \multirow[b]{2}{*}{ Mar } & \multirow[b]{2}{*}{ Abr } & \multirow[b]{2}{*}{ Mai } & \multirow[b]{2}{*}{ Jun } & \multirow[b]{2}{*}{ Jul } & \multirow[b]{2}{*}{ Ago } & \multirow[b]{2}{*}{ Set } & \multirow[b]{2}{*}{ Out } & \multirow[b]{2}{*}{ Nov } & \multirow[b]{2}{*}{ Dez } & \multirow{2}{*}{$\begin{array}{c}\text { Total } \\
\text { de } \\
\text { casos }\end{array}$} & \multicolumn{2}{|c|}{ Incidência anual } \\
\hline & & & & & & & & & & & & & & Valor & Classificação \\
\hline 2010 & 108 & 287 & 357 & 1.241 & 2.610 & 2.481 & 2.647 & 2.027 & 1.202 & 918 & 723 & 223 & 14.824 & $1.589,5$ & Epidemia \\
\hline 2011 & 186 & 228 & 439 & 309 & 283 & 211 & 183 & 194 & 238 & 155 & 68 & 214 & 2708 & 287,1 & Média \\
\hline 2012 & 207 & 323 & 1312 & 2333 & 2738 & 1911 & 1911 & 1574 & 873 & 503 & 462 & 131 & 14278 & 1497,6 & Epidemia \\
\hline 2013 & 125 & 187 & 305 & 298 & 985 & 560 & 388 & 263 & 212 & 118 & 136 & 116 & 3693 & 370,5 & Alta \\
\hline 2014 & 170 & 150 & 217 & 308 & 674 & 1013 & 1107 & 792 & 492 & 359 & 238 & 153 & 5673 & 564,3 & Alta \\
\hline 2015 & 145 & 142 & 199 & 377 & 571 & 696 & 739 & 205 & 150 & 84 & 92 & 78 & 3478 & 343,1 & Alta \\
\hline 2016 & 149 & 565 & 621 & 755 & 663 & 332 & 200 & 58 & 35 & 26 & 12 & 24 & 3440 & 336,7 & Alta \\
\hline $\begin{array}{c}\text { Total de } \\
\text { casos }\end{array}$ & 1.090 & 1.882 & 3.450 & 5.621 & 8.524 & 7.204 & 7.175 & 5.113 & 3.202 & 2.163 & 1.731 & 939 & 48.094 & - & - \\
\hline
\end{tabular}

Fonte: SMS, 2018. Elaborada pelos autores (2019).

Levando-se em consideração a população total de habitantes, a região apresentou um coeficiente de incidência anual médio de 737,12 para um grupo de 100.000 habitantes. Entre todos os anos, o ano de 2010 apresentou o maior número de notificações de casos confirmados (14.824), resultando na mais alta taxa de incidência do período analisado (1.589,5/100 mil hab). O ano de 2011 foi aquele que apresentou o menor número de casos, somando 2.708 
notificações e incidência anua calculada em 287,1/100 mil hab. A incidência de dengue na população oscilou na seguinte ordem: declínio, aumento, declínio, aumento, declínio e declínio, durante os períodos compreendidos entre 20102011, 2011-2012, 2012-2013，2013-2014，2014-2015，2015-2016, respectivamente. O número absoluto de casos de dengue por ano, com seu respectivo coeficiente de incidência, pode ser observado mediante análise da Tabela 1.

Tabela 2 - Casos de dengue por faixa etária no Município de Maceió-AL. Brasil, 2010 a 2016.

\begin{tabular}{|c|c|c|c|c|c|c|c|c|c|c|c|c|c|c|}
\hline \multirow{2}{*}{ Ano } & \multicolumn{12}{|c|}{ Faixa etária } & \multirow{2}{*}{$\begin{array}{c}\text { Total } \\
\text { casos/ano }\end{array}$} & \multirow{2}{*}{$(\%)$} \\
\hline & $<1$ & $1-4 a$ & $5-9 a$ & $10-14$ & $15-19$ & $20-29$ & 30-39 & $40-49$ & 50-59 & 60-69 & 70-79 & $80 \mathrm{e}+$ & & \\
\hline 2010 & 341 & 798 & 1.414 & 1.863 & 1.858 & 3.439 & 2.289 & 1.459 & 883 & 316 & 127 & 34 & 14.821 & 30.8 \\
\hline 2011 & 55 & 95 & 149 & 209 & 356 & 744 & 505 & 309 & 177 & 66 & 32 & 11 & 2.708 & 5.6 \\
\hline 2012 & 186 & 340 & 584 & 1207 & 1737 & 3542 & 2869 & 1896 & 1202 & 484 & 166 & 60 & 14.273 & 29.7 \\
\hline 2013 & 75 & 223 & 250 & 288 & 434 & 982 & 671 & 410 & 239 & 80 & 24 & 16 & 3.692 & 7.7 \\
\hline 2014 & 162 & 144 & 218 & 436 & 711 & 1521 & 1114 & 718 & 417 & 162 & 58 & 12 & 5.673 & 11.8 \\
\hline 2015 & 87 & 107 & 138 & 256 & 330 & 833 & 746 & 503 & 289 & 136 & 37 & 16 & 3.478 & 7.2 \\
\hline 2016 & 58 & 92 & 166 & 265 & 278 & 689 & 613 & 501 & 364 & 239 & 121 & 54 & 3.440 & 7.2 \\
\hline Total de casos & 964 & 1799 & 2919 & 4524 & 5704 & 11750 & 8807 & 5796 & 3571 & 1483 & 565 & 203 & 48.085 & 100 \\
\hline (\%) fx. etária & 2.0 & 3.7 & 6.1 & 9.4 & 11.9 & 24.4 & 18.3 & 12.1 & 7.4 & 3.1 & 1.2 & 0.4 & - & - \\
\hline
\end{tabular}

Fonte: SMS, 2018. Elaborada pelos autores (2019).

Verificou-se que a faixa etária de 20 a 29 anos foi a mais acometida, concentrando aproximadamente $1 / 4$ de todos os casos de dengue confirmados (Tabela 2).

Constatou-se que $55 \%$ dos casos ocorreram no sexo feminino, em pacientes da raça/cor parda $(35,5 \%)$ e, em relação à escolaridade, o maior percentual de dengue foi observado para a população com ensino fundamental incompleto (Tabela 3).

Tabela 3 - Características sociodemográficas dos casos de dengue no Município de Maceió-AL. Brasil, 2010 a 2016. 


\begin{tabular}{llcc}
\hline \multicolumn{1}{c}{ Característica } & $\mathbf{N}$ & $\%$ \\
\hline Sexo & & \\
& Masculino & 21.584 & 44,9 \\
& Feminino & 26.422 & 54,9 \\
$\quad$ Ignorado /Branco & 88 & 0,2 \\
Raça/Cor & & \\
Branca & 3.616 & 7,5 \\
Preta & 2.533 & 5,3 \\
Amarela & 137 & 0,3 \\
Parda & 17.075 & 35,5 \\
Indígena & 185 & 0,4 \\
Ignorado /Branco & 24.548 & 51,0 \\
Escolaridade & & \\
Analfabeto & 233 & 0,5 \\
EF incompleto & 5.984 & 12,4 \\
EF completo & 844 & 1,8 \\
EM incompleto & 1.400 & 2,9 \\
EM completo & 2.789 & 5,8 \\
ES incompleta & 437 & 0,9 \\
ES completa & 550 & 1,1 \\
Ignorado /Branco & 35.857 & 74,6 \\
\hline Total & 48.094 & 100 \\
\hline
\end{tabular}

Fonte: SMS, 2018. Elaborada pelos autores (2019).

Observou-se crescimento do número de casos ao final do primeiro semestre de cada ano, coincidindo com o período chuvoso, com a maior incidência ocorrendo entre os meses de maio e julho (Figura 1).

Quadro 1 - Coeficiente de correlação de Pearson da Taxa Mensal de Incidência de Dengue (2010-2016) em Maceió com parâmetros climáticos do Instituto Nacional de Meteorologia (INMET)

\begin{tabular}{lcccccccc}
\hline \multicolumn{1}{c}{ Variável climática } & \multicolumn{9}{c}{ Ano } & \multicolumn{2}{c}{} \\
\cline { 2 - 8 } & $\mathbf{2 0 1 0}$ & $\mathbf{2 0 1 1}$ & $\mathbf{2 0 1 2}$ & $\mathbf{2 0 1 3}$ & $\mathbf{2 0 1 4}$ & $\mathbf{2 0 1 5}$ & $\mathbf{2 0 1 6}$ \\
\hline Precipitação pluviométrica & 0,52 & 0,14 & 0,37 & 0,55 & 0,56 & 0,86 & 0,64 \\
Temperatura mensal média & $-0,69$ & 0,41 & $-0,43$ & $-0,20$ & $-0,86$ & $-0,44$ & 0,21 \\
Umidade relativa do ar & 0,90 & 0,02 & 0,56 & 0,36 & 0,90 & 0,69 & 0,17 \\
\hline
\end{tabular}

R: Coeficiente de correlação de Pearson ( $R$ de Pearson). ${ }^{*} p<0,05$. Elaborada pelos autores (2019).

Houve, ao longo dos anos, uma correlação positiva com a precipitação pluviométrica em $2010(r=0,52), 2011(r=0,14), 2012(r=0,37), 2013(r=0,55)$, $2014(r=0,56), 2015(r=0,86)$ e $2016(r=0,64)$. 


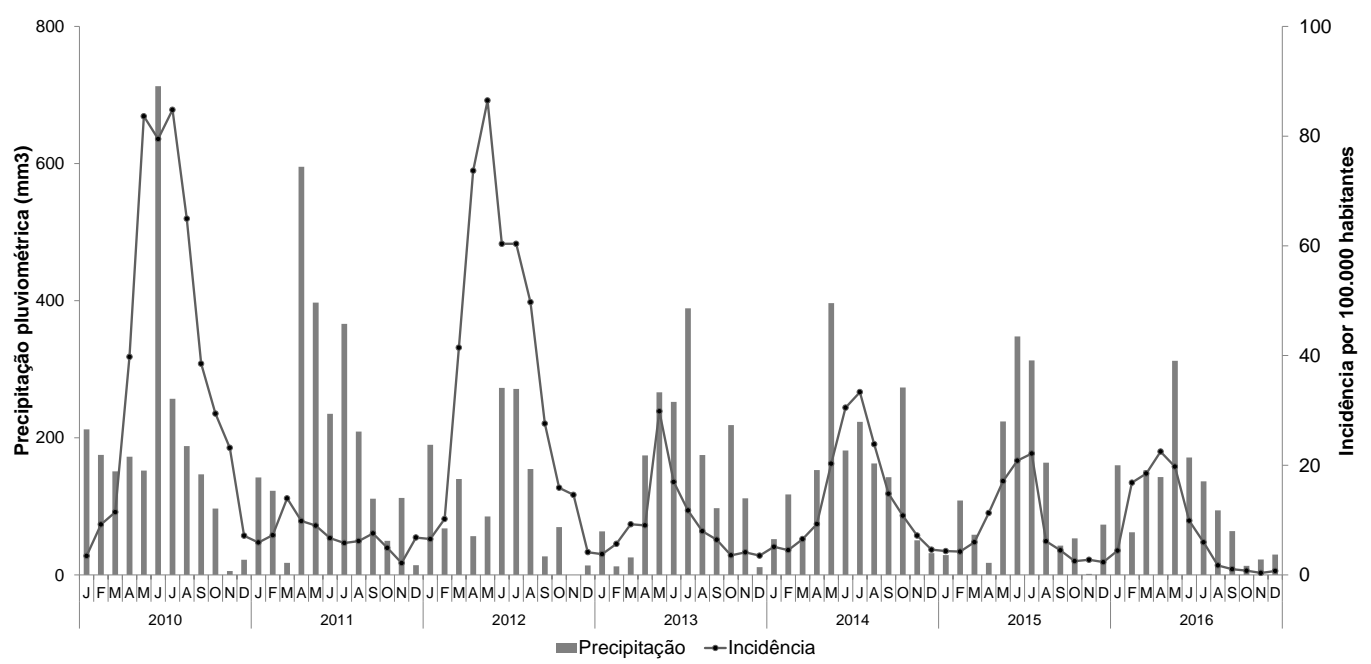

Figura 1 - Série histórica da precipitação pluviométrica mensal (mm3) e incidência de dengue mensal (100 mil habitantes) no Município de Maceió-AL. Brasil, 2010 a 2016.

Houve correlação positivo-negativa com a temperatura mensal média em $2010(r=-0,69), 2011(r=0,41), 2012(r=-0,43), 2013(r=-0,20), 2014(r=-$ $0,86), 2015(r=-0,44)$ e $2016(r=0,21)$. Igualmente, houve correlação positivonegativa com a umidade relativa do ar em $2010(r=0,90), 2011(r=0,02), 2012$ $(r=0,56), 2013(r=0,36), 2014(r=0,90), 2015(r=0,69)$ e $2016(r=-0,17)$.
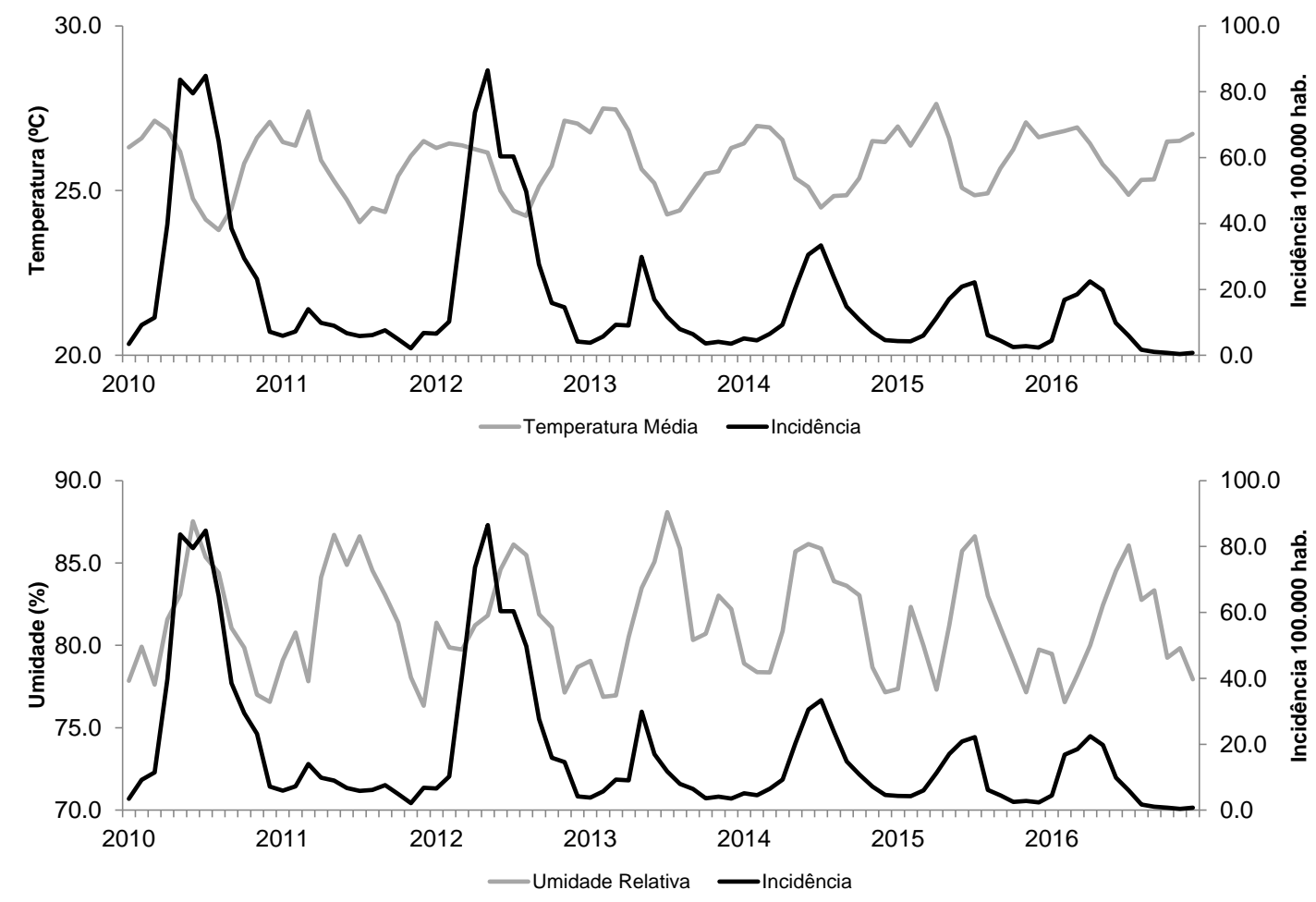

Figura 2 - Série histórica da incidência mensal de dengue e variação de dados climáticos no Município de Maceió-AL. Brasil, 2010 a 2016; (2A) Temperatura Média em º ; (2B) Umidade Relativa em \%. 
Verificou-se que nenhum bairro da cidade de Maceió apresentou ausência da doença no período de 2010-2016. As áreas consideradas de "média incidência" se distribuíram ao longo de todo território da capital, enquanto que as de "alta incidência" encontravam-se concentradas, principalmente, nas regiões Sul, Noroeste e Norte da cidade. O município apresentou somente dois bairros com "baixa incidência" (Figura 3).

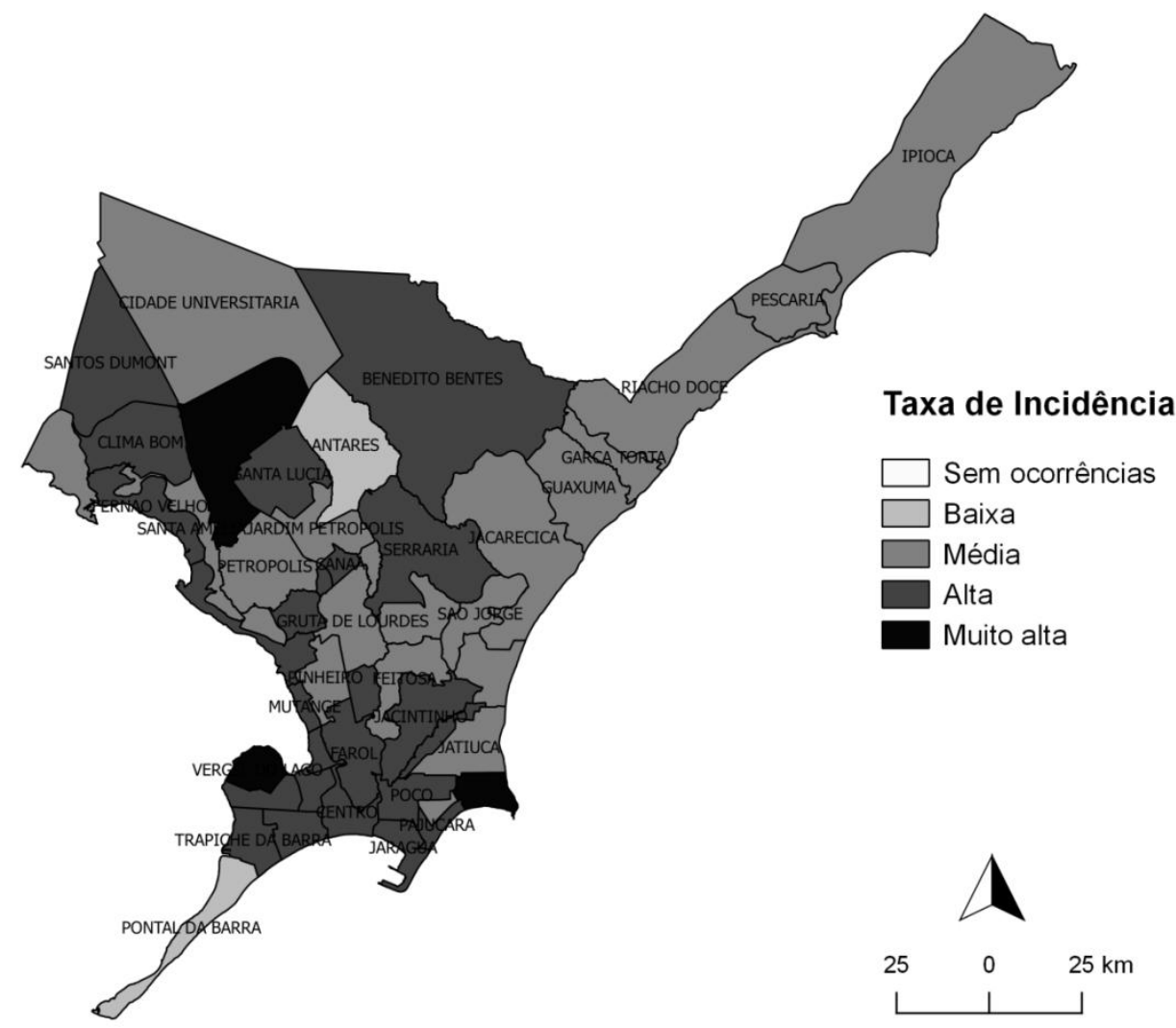

Figura 3 - Distribuição espacial do coeficiente anual médio da incidência de dengue, por bairro, em Maceió-AL, 2010 a 2016.

Ao aplicar o estimador de densidade de Kernel, por meio do método de interpolação, verificou-se alta densidade de incidência da doença (hot spot) em três regiões do município, evidenciando grande concentração de ocorrência (clusteres) de dengue principalmente na porção Sul e no Litoral da cidade (Figura 4). Tais aglomerados abrangem, especialmente, os bairros (1) Ponta Verde, Ponta da Terra, Pajuçara, Poço e Jatiúca; (2) Prado, Ponta Grossa, Vergel do Lago, Centro, Trapiche da Barra, Levada e Farol; (3) Bebedouro, Chã de Bebedouro, Chã da Jaqueira, Santo Amaro, Petrópolis e Canãa (Figura 4). 


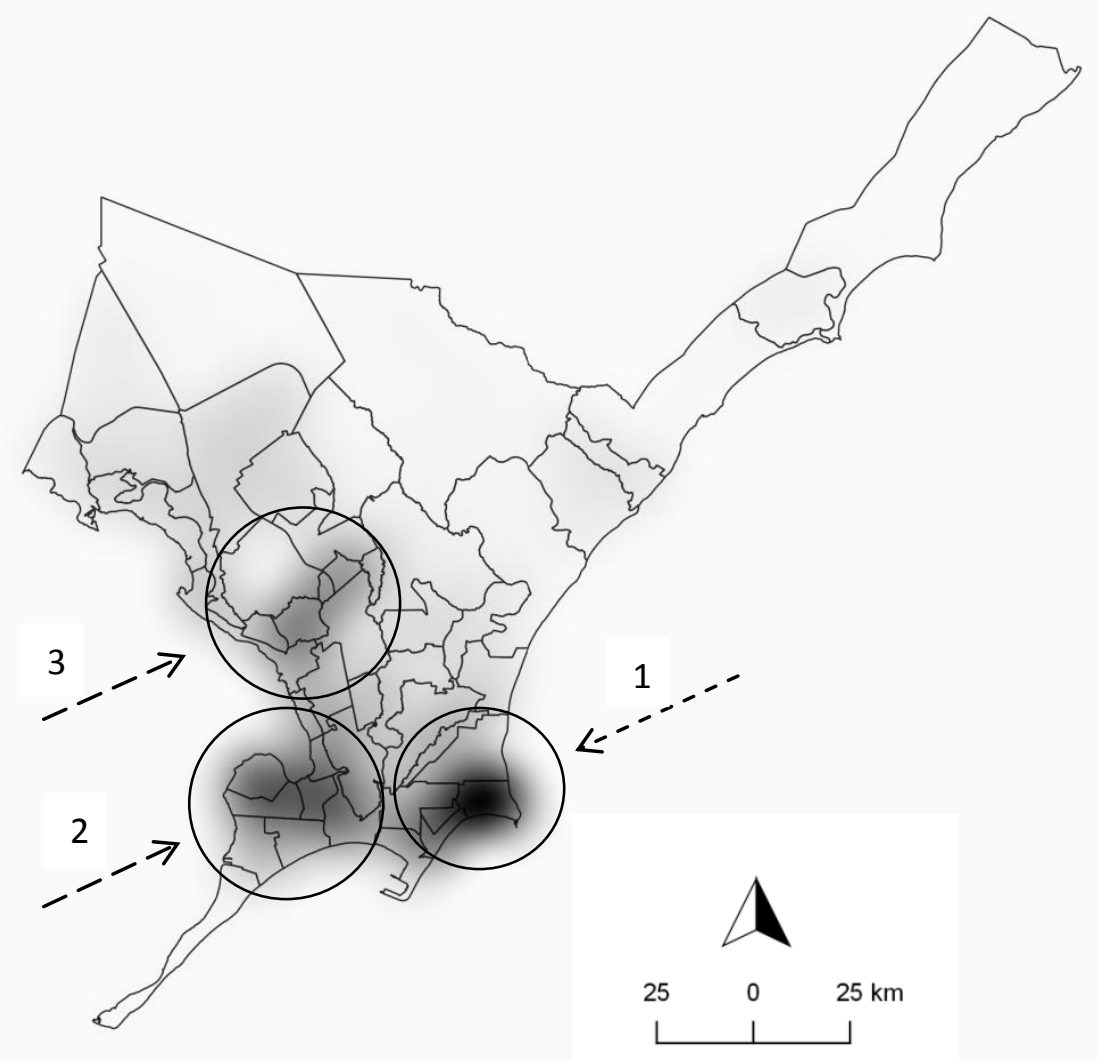

Figura 4 - Distribuição espacial da incidência de dengue, por bairro, em Maceió-AL, 2010 a 2016, utilizando estimador de densidade de Kernel.

\section{DISCUSSÃO}

A investigação da distribuição temporal de casos de dengue na cidade indicou que o primeiro triênio da pesquisa (2010-2012) concentrou o maior número de ocorrências, representando aproximadamente $65 \%$ do total de casos do presente estudo. No quadriênio (2013-2016) houve uma sensível redução na quantidade de diagnósticos, e, com efeito, nota-se uma importante queda no coeficiente de incidência.

No período de $2010-2016$ apenas 2 bairros (4\%) figuraram no grupo de baixa incidência, 21 (42\%) registraram média incidência, 24 (48\%) alta incidência e 3 (6\%) apresentaram estratificação de casos compatível com epidemia (altíssima incidência).

Nesse estudo, a dengue acometeu principalmente indivíduos pardos, do gênero feminino e em faixa etária reprodutiva. Esse perfil é concordante com as características da doença relatadas em outros estados do Brasil e do Nordeste (MINISTÉRIO DA SAÚDE, 2019; SILVA \& MACHADO, 2018; VIANA; IGNOTTI, 2013).

Pelo uso da ferramenta de mapa temático, vimos que, de uma forma geral, a ocorrência da dengue está dispersa em Maceió. Os bairros com maior número de casos (alta incidência) situam-se, em sua maioria, na porção sul/litorânea da cidade. Ainda em termos de distribuição, verifica-se que há 
uma menor concentração da doença nos bairros do litoral norte e localizados na porção leste do município. Padrão espacial semelhante também fora identificado nas cidades de Natal e Fortaleza em estudo desenvolvido por Silva \& Machado (2018) que analisou a associação da dengue com variáveis sócioespaciais no Nordeste brasileiro.

O estimador Kernel é uma técnica de interpolação exploratória que gera uma superfície de densidade para a identificação visual de áreas quentes, as quais apresentam-se com concentração de eventos que indicam a aglomeração em uma distribuição espacial(HORTA et al., 2014). Neste trabalho, a utilização da referida ferramenta indicou que não há um comportamento sistemático de agrupamento no território, tendendo os indicadores à irregularidade espacial.

A estimativa de áreas sujeitas a maior ocorrência de riscos de casos de dengue através da metodologia de interpolação de Kernel revelou também que há pelo menos três áreas que mais se destacaram no estudo, sendo a área mais crítica àquela situada nas imediações do bairro de Ponta Verde, região de maior densidade na identificação visual de áreas quentes. Na tentativa de explicar essa distribuição em considerando seu recorte espacial, Pereira et al. (2014) afirmam que contribuem severamente para a proliferação do vetor transmissor, e por conseguinte, para maior ocorrência da doença, a grande circulação de pessoas e de mercadorias, o adensamento populacional e de edificações, problemas de infraestrutura, presença de canteiros de obras, existência de terrenos baldios e de imóveis sem utilização.

Verifica-se que os coeficientes de incidência de dengue em Maceió-AL foram maiores nos meses de maior pluviosidade e de elevados índices de umidade relativa do ar, indicando que há importância dessas variáveis na maior proliferação do vetor. Da mesma forma, percebeu-se que a temperatura média foi maior nos períodos que precediam os meses de acentuado número de casos de dengue confirmados.

As análises estatísticas de correlação entre a incidência de dengue e as variáveis umidade relativa do ar e precipitação pluviométrica evidenciaram correlação de forte a moderada, respectivamente em $60 \%$ e $70 \%$ do período analisado. Para os valores mensais de temperatura média, por sua vez, a análise quantitativa resultou em coeficientes de correlação fracos ou desprezíveis para todo o período.

Os achados desse estudo foram consistentes com trabalho realizado por Rebelo et al. (1999) que, ao investigar a distribuição do Aedes aegypti e os casos de dengue durante 10 anos em 87 municípios do Nordeste, verificou que os casos notificados da doença e o índice de infestação predial por Aedes foram maiores nos meses úmidos. Outros estudos mais recentes desenvolvidos em diversas regiões do Brasil também identificaram que houve maior incidência de dengue e de infestação predial no primeiro semestre de cada ano, coincidindo com o período de elevada pluviosidade e de maiores percentuais de umidade do ar. A transmissão da doença reduziu nos meses de julho a setembro, época de poucas chuvas (CODEÇO et al., 2009; SOUZA et al., 2010; PEREIRA et al., 2010; ROSEGHIN et al., 2011; PEREDA et al., 2011; MAGALHÃES; ZANELLA, 2013; ELY, 2014; DE SOUSA PAIVA et al. 2015; (FERNANDES et al., 2012; FERREIRA et al., 2018; VIANA; IGNOTTI, 2013). Tais comportamentos segundo Viana e Ignotti (2013) ocorrem, pois os fatores climáticos influem diretamente na dinâmica de reprodução, distribuição e proliferação do vetor e na formação 
de seus criadouros. Horta et al. (2014) destacam, sobre esse tema, que modelos com base em variáveis meteorológicas podem ser úteis em programas de controle de dengue em países tropicais.

Baseado nos resultados apresentados, pode-se inferir que existe relação entre a média da precipitação e a quantidade de casos de dengue confirmados, corroborando com as conclusões de Honório (2009) de que "quando as chuvas acontecem, de 3 a 5 semanas após há um efeito sobre a densidade de vetores e consequente maior número de casos da doença". Para Ferreira et al. (2018), esse fenômeno explica-se em razão da pluviosidade e da temperatura elevadas, que favorecem o aumento do número de criadouros e, consequentemente, de casos de dengue. Ainda em conformidade com os autores, em períodos secos e de baixas temperaturas, há diminuição no número de vetores, mas tal fator sozinho não é suficiente para cessar a transmissão da doença.

Este estudo pode conter limitações, incluindo possíveis vieses relacionados aos procedimentos de amostragem e ao desenho metodológico escolhido. A escala de dados, por contemplar um período de sete anos, foi recrutada para a média mensal para cada uma das variáveis climáticometeorológicas, o que pode explicar a variabilidade de resultado para as correlações expressas no capítulo "resultados". As análises de correlação não foram desenvolvidas, a priori, com a finalidade de predição de valores ou de realizar a inferência da participação de múltiplas variáveis na explicação da ocorrência da Dengue. Adotou-se, aqui, uma medida de associação bivariada do grau de relacionamento entre duas variáveis. Além disso, por tartar-se de estudo ecológico, destaca-se a possibilidade de confundimento residual devido à ausência de controle das associações por variáveis não disponíveis nas bases de dados, além das dificuldades de definição da temporalidade entre os eventos analisados e em atribuir ao nível individual às mesmas associações ecológicas aqui identificadas. Assim, é plausível inferir que, devido às particularidades inerentes aos métodos adotados, a concordância dos dados apresentados neste trabalho podem divergir daquela relatada pela literatura em relação ao mesmo fenômeno analisado. Porém, mesmo com essas considerações, é importante salientar que que tais dados são oriundos de bases de dados oficiais de órgãos públicos especializados e que, inclusive, adotam essas informaçoes como medida de referência para tomada de decisões em relação à adoção de políticas públicas.

\section{CONCLUSÃO}

Na estratificação espacial da TAM, o município de Maceió apresentou $57 \%$ do período como alta incidência e $29 \%$ do intervalo com característica de epidemia. Nenhum bairro da cidade de Maceió apresentou ausência da doença na totalidade período em análise, sendo que as áreas consideradas de média incidência se distribuíram ao longo de todo território da capital, enquanto que as de alta incidência encontravam-se concentradas, principalmente, nas regiões Sul, Noroeste e Norte da cidade. Na análise de densidade de casos, verificou-se alta densidade e formação clusteres em três regiões do município e grande concentração de ocorrência de dengue nas porções Sul e no Litoral da cidade. Houve correlação de forte a moderada entre a incidência de dengue e as variáveis umidade e precipitação pluviométrica em $60 \%$ e $70 \%$ do período analisado, respectivamente. O perfil de acometidos é compatível com a 
literatura e foi expresso por maioria de acometidos jovens-adultos, do sexo feminino, de raça/cor parda, com baixa escolaridade. Os resultados da pesquisa apontam que as variáveis climáticas (precipitação e umidade) influenciam na epidemiologia da dengue, além de evidenciarem a existência de áreas prioritárias de intervenção no combate a transmissão da doença.

Outros estudos, com emprego de técnicas multivariadas, de maior poder explicativo e com o uso de variadas escalas tempo-espaciais devem ser incentivados, haja vista que as estratégias de avaliação da correlação entre variáveis, no campo da saúde, maximizam a compreensão do fenômeno estudado e, por conseguinte, viabilizam a atuação direcionada do Poder Público.

\section{REFERÊNCIAS}

AULT, S, et al. The Decline of Dengue in the 471 Americas in 2017: Discussion of Multiple Hypotheses. Trop Med Int Health, v. 24, n. 1, p. 1-17, 2019.

BARCELLOS, Christovam et al. Identification of places with potential transmission of dengue fever in Porto Alegre using Geographical Information Systems. Revista da Sociedade Brasileira de Medicina Tropical, v. 38, n. 3, p. 246-250, 2005.

BARRETO, Maurício L.; TEIXEIRA, Maria Glória. Dengue in Brazil: epidemiological situation and contribution to a research agenda. estudos avançados, v. 22, $\mathrm{n}$. 64, p. $53-72,2008$.

BHATT, Samir et al. A distribuição global e o ônus da dengue. Nature , v. 496, n. 7446, p. 504, 2013.

CAVALCANTE, Micheline Pimentel Ribeiro et al. Geospatial analysis: a study about dengue. Acta Paulista de Enfermagem, v. 26, n. 4, p. 360-368, 2013.

CODEÇO, Cláudia Torres et al. Seasonal dynamics of Aedes aegypti (Diptera: Culicidae) in the northernmost state of Brazil: a likely port-of-entry for dengue virus 4. Memórias do Instituto Oswaldo Cruz, v. 104, n. 4, p. 614-620, 2009.

DE SOUSA PAIVA, Paulo Roberto; BRANDÃO, Cássia Barreto; DE MIRANDA, Ricardo Augusto Calheiros. ASSOCIAÇÃO ENTRE A INCIDÊNCIA DE DENGUE E OS ELEMENTOS CLIMÁTICOS NA XVI RA DE JACAREPAGUÁ-RJ. Geoambiente On-line, n. 24, 2015.

ELY, Deise Fabiana. Tendências climáticas e a incidência da dengue em cidades do Sul do Brasil: estudo de caso de londrina, Maringá (PR) e Florianópolis (SC). Revista Brasileira de Climatologia, v. 13, 2014.

FERNANDES, Rosilainy Surubi et al. Clima e casos de dengue em Tangará da Serra/MT. Hygeia, v. 8, n. 15, 2012.

FERREIRA, Aline Chimello; CHIARAVALLOTI, Francisco; MONDINI, Adriano. Dengue em Araraquara, SP: epidemiologia, clima e infestação por Aedes aegypti. Rev. Saúde Pública, v. 52, 2018.

FIGUEIREDO FILHO, Dalson Britto; SILVA JúNIOR, José Alexandre da. Desvendando os Mistérios do Coeficiente de Correlação de Pearson ( $r$ ). Revista Política Hoje. v. 1, n. 8, p. 115-46, 2009. 
HONORATO, Taizi et al. Spatial analysis of distribution of dengue cases in Espírito Santo, Brazil, in 2010: use of Bayesian model. Revista Brasileira de Epidemiologia, v. 17, p. 150-159, 2014.

HONÓRIO, Nildimar Alves et al. The spatial distribution of Aedes aegypti and Aedes albopictus in a transition zone, Rio de Janeiro, Brazil. Cadernos de Saúde Pública, v. 25, p. 1203-1214, 2009.

HORTA, Marco Aurelio et al. Temporal relationship between environmental factors and the occurrence of dengue fever. International journal of environmental health research, v. 24, n. 5, p. 471-481, 2014.

INSTITUTO BRASILEIRO DE GEOGRAFIA E ESTATÍSTICAS. Estatísticas Populacionais 2010-2016. Available from: https://www.ibge.gov.br/.

LOPES, Thaísa Regina Rocha et al. Dengue in Brazil in 2017: what happened?. Revista do Instituto de Medicina Tropical de São Paulo, v. 60, 2018.

MAGALHÃES, Gledson Bezerra; ZANELLA, Maria Elisa Elisa. Comportamento espacial da dengue e sua relação com o clima na região metropolitana de Fortaleza. Revista Brasileira de Climatologia, v. 12, n. 1, 2013.

MINISTÉRIO DA SAÚDE (BR). Monitoramento dos casos de dengue, febre de chikungunya e febre pelo vírus Zika até a Semana Epidemiológica, v. 49, n. 9, 2019

MINISTÉRIO DA SAÚDE (MS). Secretaria de Vigilância em Saúde. Programa Nacional de Controle de Dengue. $1^{\text {a }}$ edição. Brasília: MS; 2002. 34 p.

MONTEIRO, Eridan Soares Coutinho et al. Aspectos epidemiológicos e vetoriais da dengue na cidade de Teresina, Piauí-Brasil, 2002 a 2006. Epidemiologia e Serviços de Saúde, v. 18, n. 4, p. 365-374, 2009.

PEREDA, Paula Carvalho; DE OLIVEIRA ALVES, Denisard Cneio; DE ALMEIDA RANGEL, Marcos. Elementos climáticos e incidência de dengue: teoria e evidência para municípios brasileiros. In: $33^{\circ}$ Meeting of the Brazilian Econometric Society. 2011.

PEREIRA, Carlos Alexandre Rodrigues et al. Economic evaluation of cases of dengue fever attributed to the disaster of 2011 in Nova Friburgo (State of Rio de Janeiro/Brazil). Ciencia \& saude coletiva, v. 19, n. 9, p. 3693-3704, 2014.

PEREIRA, Clarissa Cardoso; DE FÁTIMA MARIANO, Zilda; ROCHA, José Ricardo Rodrigues. DENGUE: UMA ANÁLISE CLIMATO-GEOGRÁFICA NA CIDADE DE JATAÍ-GO. Revista Brasileira de Climatologia, v. 6, 2010.

RAMOS-CASTANEDA, Jose et al. Dengue in Latin America: systematic review of molecular epidemiological trends. PLoS neglected tropical diseases, v. 11, n. 1, p. e0005224, 2017.

REBÊLO, José Manuel Macário et al. Distribuição de Aedes aegypti e do dengue no Estado do Maranhão, Brasil. Cadernos de Saúde Pública, v. 15, p. 477-486, 1999.

RODRIGUES, V. P. Conceitos e ferramentas de epidemiologia. $1^{\text {a }}$ edição. São Luís: UFMA; 2014. 13 p.

ROSEGHINI, WFF et al. Dengue epidemics in middle-south of Brazil: Climate constraints and some social aspects. Revista Brasileira de Climatologia, v. 9, 2011. 
SILVA, JULIO CESAR BARRETO DA; MACHADO, CARLOS JOSÉ SALDANHA. Associations between dengue And socio-environmentAl vAriAbles in CApitAls of the brAziliAn northeAst by cluster AnAlysis. Ambiente \& Sociedade, v. 21, 2018.

SOUZA, Sócrates Siqueira de; SILVA, Ionizete Garcia da; SILVA, Heloísa Helena Garcia da. Associação entre incidência de dengue, pluviosidade e densidade larvária de Aedes aegypti, no Estado de Goiás. Rev Soc Bras Med Trop, p. 152155, 2010.

VIANA, Dione Viero; IGNOTTI, Eliane. A ocorrência da dengue e variações meteorológicas no Brasil: revisão sistemática. Revista Brasileira de Epidemiologia, v. 16, p. 240-256, 2013.

WORLD HEALTH ORGANIZATION (WHO). Global strategy for dengue prevention and control. Geneva: WHO; 2012. 43 p 